\title{
Efficacy and Enzymatic Synthesis of Prebiotic Galacto-Oligosaccharides from Lactose Against Colon Carcinogenesis in Male Rats
}

\author{
Naureen Naeem ${ }^{1 *}$, Shahid Raza ${ }^{1}$, Hira Mubeen ${ }^{2}$, Shoaib Ahmad Siddiqui ${ }^{3}$ and Raana Khokhar ${ }^{4}$ \\ ${ }^{1}$ Department of Home-Economics, Lahore Garrison University, Lahore, Pakistan \\ ${ }^{2}$ Department of Computer Science, Lahore Garrison University, Lahore, Pakistan \\ ${ }^{3}$ Department of Biology, Lahore Garrison University, Lahore, Pakistan \\ ${ }^{4}$ Department of Food Science and Human Nutrition, University of Veterinary and Animal Sciences, Lahore, Pakistan
}

*Corresponding author: Naureen Naeem, Department of Home-Economics, Lahore Garrison University, Lahore, Pakistan, Tel: +92 42 37181821; E-mail: naureen.naeem@lgu.edu.pk

Received date: May 30, 2018; Accepted date: June 12, 2018; Published date: June 19, 2018

Copyright: (c) 2018 Naeem N, et al. This is an open-access article distributed under the terms of the Creative Commons Attribution License, which permits unrestricted use, distribution, and reproduction in any medium, provided the original author and source are credited.

\begin{abstract}
In the present study the role of galacto-oligosaccharides (GOS) was assessed against development of colon cancer in rats. This study was based on the object to synthesize prebiotic galacto-oligosaccharides from lactose and to assess the protective role of galacto-oligosaccharide against colon cancer. Research was carried out in two phases, production of prebiotic galacto-oligosaccharide and assessment of their protective role against colon cancer. Highest \%age of enzyme activity was observed at $6.5 \mathrm{pH}$ and $37^{\circ} \mathrm{C}$. Transgalactosylation was carried out and the process was carried out at $37^{\circ} \mathrm{C}$ using three different concentrations of enzyme. Animals belonging to prebiotic galacto-oligosaccharides groups (G2, G3 and G4) and inulin (Go) had significantly decreased aberrant crypt foci (ACF) counts per colon as compared to controlled animals ( $G 1$ and G5). The body mass of animals belonging to galacto-oligosaccharides (Group G2, G3 and G4) increased significantly higher as compared to control group (G1). Effect of pre-biotic treatment had significant effects on organ weights; liver, heart, spleen as well as small intestine length. In our findings galacto-oligosaccharides (GOS) were found to be protective against colon cancer however further research is needed at DNA level to find out the mutations at gene levels.
\end{abstract}

Keywords: $\quad$ Galacto-oligosaccharides; Bifidobacteria; Pre-biotics

\section{Introduction}

Functional foods exert a vital role in the prevention of nutritionrelated diseases. A food said to be functional contains new and technologically advanced ingredients having some specific health benefits [1]. Among most frequently used functional food compounds are probiotics, prebiotics, vitamins, and calcium and plant antioxidants [2]. Colon cancer is the third leading cause of cancer-related deaths in whole world and is fourth most common cancer type. Mortality rate associated with colon cancer is high because of insidious onset of this disease.

Dietary habits can either increase or decrease the risk of colon cancer. In this aspect, much importance is given to probiotics such as Lactobacilli or Bifidobacteria, and prebiotics such as fructans or fructo-oligosaccharides. As, some studies have reported that their intake is associated with less occurrence of chemically induced colon cancers in animal models. Functional foods play a vital role in preventing nutrition-related diseases. A food said to be functional contains new and technologically advanced ingredients having some specific health benefits [1]. Among, most frequently used functional food compounds are probiotics, prebiotics, vitamins, calcium and plant antioxidants [2].

Gut micro flora consists of different populations of microorganisms including harmful as well as health promoting microorganisms. A wide variety of interactions take place between these microorganisms. In order to improve colonic health, minimize the risk of various diseases and well-being of host, it is necessary to achieve a "balanced gut micro flora". Various dietary strategies could be implied to modify composition of colonic microbiota [3]. More recently, prebiotics are being used as a strategy for obtaining balanced microflora. These are non-digestible ingredient which positively affects the host by selective stimulation of activity or growth of one or limited number of colonic bacteria thereby improving host health" [4]. Colon cancer is the third leading reason of cancer-related deaths in whole world and is fourth most common cancer type. Mortality rate associated with colon cancer is high because of insidious onset of this disease. Dietary habits can either increase or decrease the risk of colon cancer. In this aspect, much importance is given to probiotics such as Lactobacilli or Bifidobacteria, and prebiotics such as fructans or fructo-oligosaccharides.

Several studies concluded that galacto-oligosaccharides (GOS) help promote immunity by exhibiting inhibitory effect on enteric pathogenic infections. They cause maximum decrease in harmful Clostridiaascompared to another pre-biotics [5]. Galactooligosaccharides (GOS) exhibit various beneficial effects on human health. Other than maintaining a balanced micro flora, they also enhance digestibility of dairy products and lactose tolerance, reduction in serum cholesterol levels, risk of cancers, enhance mineral absorption [6]. Aim of present research was to find out effect of prebiotic treatment on the onset of colon cancer. 
Citation: $\quad$ Naeem N, Raza S, Mubeen H, Siddiqui SA, Khokhar R (2018) Efficacy and Enzymatic Synthesis of Prebiotic Galacto-Oligosaccharides from Lactose Against Colon Carcinogenesis in Male Rats. J Bioprocess Biotech 8: 330. doi:10.4172/2155-9821.1000330

Page 2 of 5

\section{Materials and Methods}

\section{Enzyme production}

Escherichia coli (E. coli) containing beta-galactosidase gene from Lactobacillus reuteri L103 was cultured as the source of the $\beta$ galactosidase enzyme. E. coli cells were grown on Luria-Bertani broth (LB) containing the appropriate antibiotics $(100 \mu \mathrm{g} / \mathrm{mL}$ ampicillin) required for maintaining the plasmids. Bacteria was grown at $37^{\circ} \mathrm{C}$ in incubator for 14-16 hours in $5 \mathrm{ml} \mathrm{LB}$ medium, then 1-5\% of this culture was transferred into $40 \mathrm{ml}$ medium and was grown again at $37^{\circ} \mathrm{C}$ for $14-16$ hours. $1-5 \%$ of this culture was transferred into $2000 \mathrm{ml}$ medium and was grown again at $37^{\circ} \mathrm{C}$ until optical density of this media was between $0.3-0.6$, then isopropyl- $\beta$-D-thiogalactoside (IPTG) was added (final conc. in media $0.1 \mathrm{mM}$ ), after adding IPTG, bacteria was grown at $25^{\circ} \mathrm{C}$ for 16 hours.

\section{Enzyme assays}

After enzyme isolation $\beta$-Galactosidase $(\beta$-gal) activity was determined using $\mathrm{O}$-nitrophenyl- $\beta$-D-galactopyranoside (ONPG) as the substrates. $20 \mu \mathrm{L}$ enzyme sample was added to $480 \mu \mathrm{L}$ of $22 \mathrm{mM}$ ONPG in buffer (50 mM sodium phosphate buffer, $\mathrm{pH}$ 6.5) (Figure 1). After 10 minutes reaction was stopped by adding $750 \mu \mathrm{L} \mathrm{Na}_{2} \mathrm{CO}_{3}(0.4$ $\mathrm{M})$. The release of o-nitrophenol was assessed by measuring the absorbance at $420 \mathrm{~nm}$.

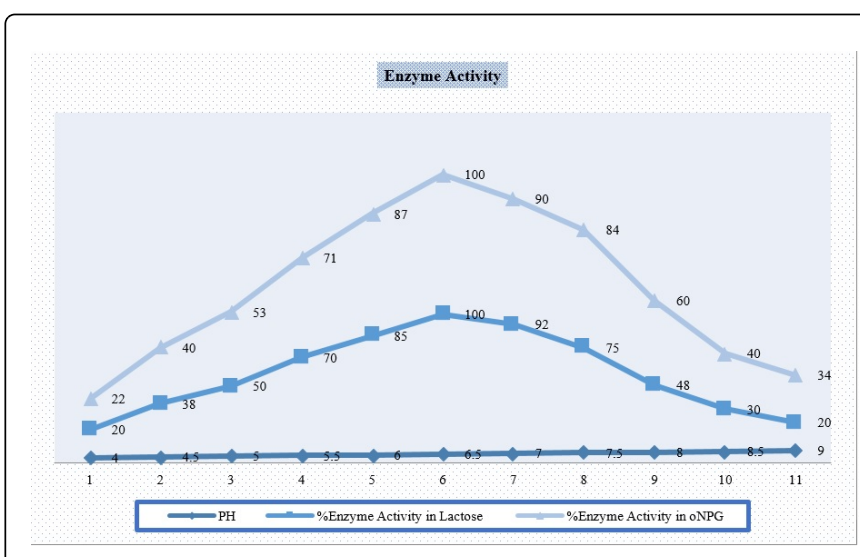

Figure 1: Effect of $\mathrm{pH}$ on $\beta$-Galactosidase activity.

\section{Thin layer chromatography (TLC)}

After transgalactosylation process the samples were used for thin layer chromatography and for the quantification of galactooligosaccharides. The samples were diluted in the ratio of $1: 9$ by taking $20 \mu \mathrm{l}$ of the sample and $180 \mu \mathrm{l}$ of distilled $\mathrm{H}_{2} \mathrm{O}$ and mixed properly before using for the TLC. $1.0 \mu \mathrm{l}$ from each sample along with standards (glucose, galactose, lactose) was taken and applied on silica gel plate. Running buffer used in TLC was prepared by water, n-propanol, ethanol, and n-butanol in the ratio of 2:3:3:2 respectively. Silica gel plates were put in the chromatographic tank till the buffer reached the $2 / 3 \mathrm{rd}$ of the plate at the upper end. TLC was run for 45 minutes. Now the plates were taken out of the chromatographic tank and dried in the hot air oven at $100^{\circ} \mathrm{C}$. Staining buffer $\left(0.6 \mathrm{~g}\right.$ thymol $+5 \mathrm{ml} \mathrm{H}_{2} \mathrm{SO}_{4}(95 \%)$ $+95 \mathrm{ml}$ ethanol) was sprayed on the dried plates. Sugar stains were visible after $110^{\circ} \mathrm{C}$ heating in hot air oven.

\section{Quantification of galacto-oligosaccharides}

Quantification of Galacto-oligosaccharides was done by measuring lactose, D-galactose and D-glucose through Megazyme kits (Megazyme International, Ireland).

\section{Calculation of galacto-oligosaccharide}

Total Galacto-oligosaccharides produced were calculated by following formula:

Concentration of GOS $(\mathrm{g} / \mathrm{L})=\mathrm{A}-\mathrm{B}+\mathrm{C}+\mathrm{D}$

$\mathrm{A}=$ Initial lactose concentration $(\mathrm{g} / \mathrm{L})$;

$\mathrm{B}=$ Lactose concentration after transgalactosylation $(\mathrm{g} / \mathrm{L})$;

$\mathrm{C}=$ Galactose concentration a ter transgalactosylation $(\mathrm{g} / \mathrm{L})$;

$\mathrm{D}=$ Glucose concentration a ter transgalactosylation $(\mathrm{g} / \mathrm{L})$.

\section{Efficacy studies}

For efficacy study 54 male Sprague Dawley rats weighing 135-190 g were used. Approval was taken from Ethical Committee of University of Veterinary and Animal Sciences, Lahore. Rats were housed under 12-hour light-dark cycles, $55 \pm 5^{\circ} \mathrm{C}$ relative humidity and temperature $23 \pm 2^{\circ} \mathrm{C}$ (Figure 2). Water and standard pellet diet were given.

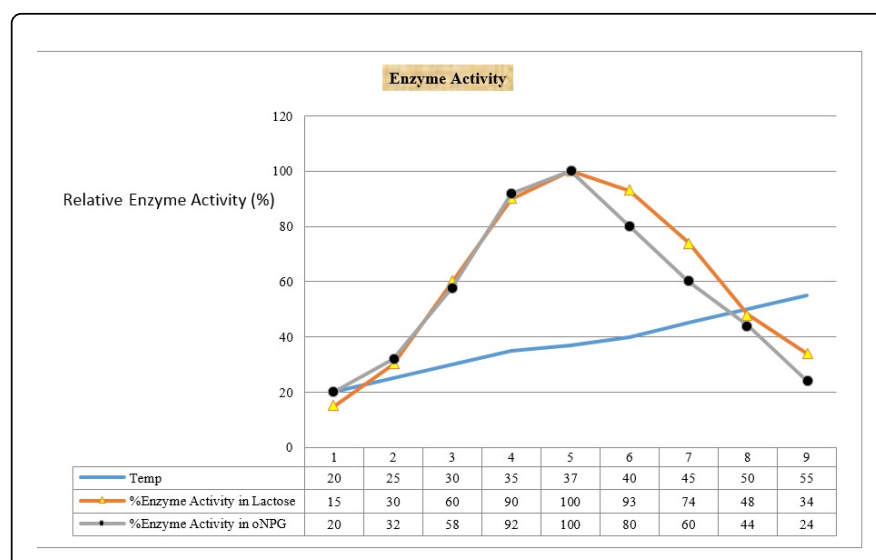

Figure 2: Effect of temperature on $\beta$-Galactosidase activity.

Induction of colon cancer: Cancer was induced in all rats by using 1 , 2-Dimethylhydrazine dihydrochloride (DMH). DMH was prepared in $1 \mathrm{mM}$ EDTA and $\mathrm{pH}$ was set at 7.0 using $1 \mathrm{mMNaOH}$. DMH was injected subcutaneously at a dose of $40 \mathrm{mg} / \mathrm{kg}$ body weight twice a week for two weeks [7] (Figures 3 and 4).

Experimental design: After receiving last dose of DMH, rats were randomly divided into six groups, one positive control group (G0), one negative control group (G1), three treatment groups (G2, G3, G4) and one control group (G5). Each group consisted of nine rats and they were kept in separate cages. Before any experimental intervention, all rats were fed on standard basal diet for a period of one week. Negative control group (G1) was fed on standard basal diet alone. Positive control group (G0) was fed on inulin ( $84 \mathrm{mg} / 100 \mathrm{~g}$ body weight), while treatment groups G2, G3 and G4 received $60 \mathrm{mg}, 84 \mathrm{mg}$ and 107.14 $\mathrm{mg} / 100 \mathrm{~g}$ body weight respectively of GOS along with standard diet for a period of 60 days. Nasogastric feeding tube was used for administration of GOS and inulin. Three rats from each group were 
Citation: $\quad$ Naeem N, Raza S, Mubeen H, Siddiqui SA, Khokhar R (2018) Efficacy and Enzymatic Synthesis of Prebiotic Galacto-Oligosaccharides from Lactose Against Colon Carcinogenesis in Male Rats. J Bioprocess Biotech 8: 330. doi:10.4172/2155-9821.1000330

Page 3 of 5

decapitated at 30 and 60 days of trial for assessment of aberrant crypt foci (ACF), liver and kidney function tests and organ to body weight ratio.

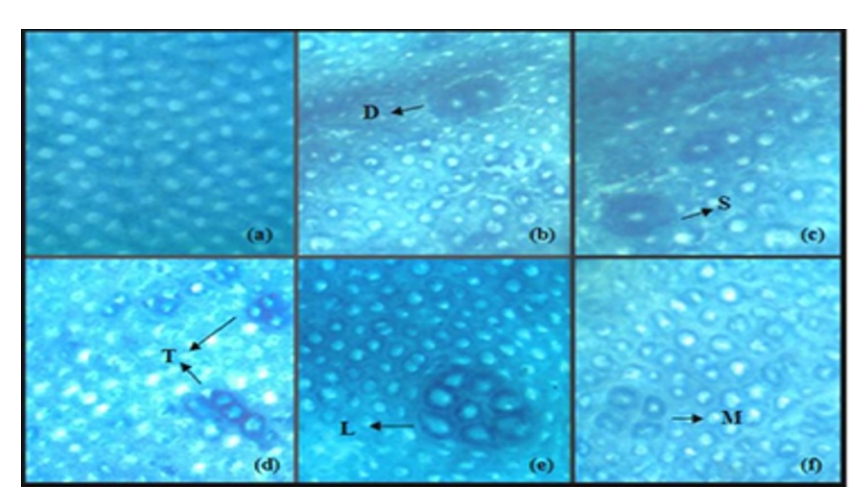

Figure 3: Topographic view of colon. Showing: (a) normal crypts of control rats, (b-f) DMH Showing: (a) normal crypts of control rats, (b-f) DMH treated rats showing ACF (aberrant crypt foci) after staining with methylene blue. Arrows indicate ACF: S-single, Ddouble, T-triplet, L-large, M-Medium.

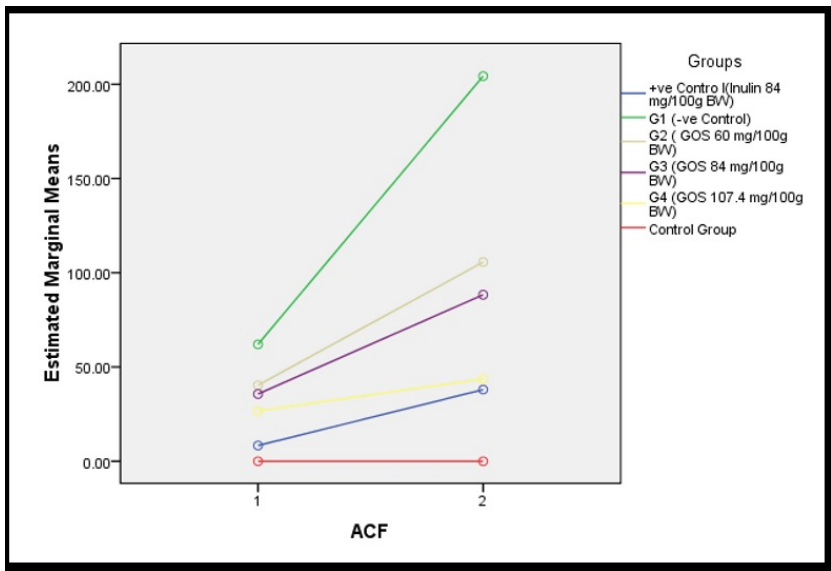

Figure 4: Topographic view of colon showing: (a) normal crypts of control rats, (b-f) DMH treated rats showing ACF (aberrant crypt foci) after staining with methylene blue. Arrows indicate ACF: Ssingle, D-double, T-triplet, L-large, M-Medium.

Organ to body weight ratio: After dissection, organs i.e., liver, kidney, heart, spleen, small intestine, large intestine was collected to evaluate the effect of test diets on different organ weights of rats. Organs were cleaned and weighed on weight balance [8]. Intestine length was measured with a measuring scale. Results were expressed as organ to body weight ratios ( $\mathrm{g} / 100 \mathrm{~g}$ of body weight).

Liver and kidney function tests (LFTs and RFTs): Serum was analyzed for liver functioning through determination of enzymes; alkaline phosphatase (ALP) and Alanine Aminotransferase (ALT). Kidney function was also evaluated through serum creatinine and urea assessment [9].

Enumeration of aberrant crypt foci (ACF): After decapitation, the colon was removed, and aberrant crypt foci were counted from the distalpart of colon which was cut into $2.5 \mathrm{~cm}$ long small sections. Section was stained using $0.2 \%$ methylene blue dye and aberrant crypt foci were counted by using light microscope. The total number of aberrant crypt foci/ rat was calculated as the sum of the large, medium and small aberrant crypt foci [10].

\section{Statistical analysis}

Results were analyzed by applying one-way analysis of variance (ANOVA) followed by Post Hoc LSD tests and were expressed as mean \pm standard deviation(SD). The differences among groups were considered significant when $\mathrm{p} \leq 0.05$.

\section{Results}

\section{Aberrant crypt foci count}

There was a significant decrease $(\mathrm{p}<0.05)$ in total ACF counts/colon in all prebiotic groups G0, G2, G3 and G4 as compared with G1 (DMH +basal diet). Total ACF counts in G0 group (Inulin+DMH) were significantly less as compared to galacto-oligosaccharide groups G1 (basal diet+DMH), G2 (GOS, 60 mg/100 g B.W+DMH), G3 (GOS, 84 $\mathrm{mg} / 100 \mathrm{~g}$ B.W+DMH), G4 (GOS,107.14 mg/100 g B.W+DMH). Among galacto-oligosaccharide groups, maximum reduction in total ACF counts was shown by G4 (107.14 mg/100 g body weight GOS) followed by G3 (84 mg/100 g body weight GOS) and then G2 (60 $\mathrm{mg} / 100 \mathrm{~g}$ B.W GOS).

\begin{tabular}{|c|c|c|}
\hline Groups $(n=9)$ & Total ACF Count & Std. Error \\
\hline $\mathrm{G} 0^{\text {bcdef }}$ & $7.33 \pm 1.52^{*}$ & 0.88192 \\
\hline $\mathrm{G} 1^{\text {acdef }}$ & $59.33 \pm 1.52$ & 0.88192 \\
\hline G2 $2^{\text {abdef }}$ & $48.00 \pm 1.00$ & 0.57735 \\
\hline G3abcef & $44.66 \pm 1.52$ & 0.88192 \\
\hline G4 $4^{\text {abcdf }}$ & $35.66 \pm 1.15$ & 0.66667 \\
\hline G5 abcde & $0.00 \pm 0.00$ & 0 \\
\hline Total & 998 & 908 \\
\hline
\end{tabular}

Table 1: Effect of prebiotic treatment on total number of aberrant crypt foci $(\mathrm{ACF})$ counts. ${ }^{\star}$ Values are expressed as Mean $\pm \mathrm{SD}$. G0 $=$ Inulin $(84$ $\mathrm{mg} / 100 \mathrm{~g} \mathrm{~B} . \mathrm{W}+\mathrm{DMH}), \mathrm{G} 1=$ basal diet+DMH, G2=GOS $(60 \mathrm{mg} / 100 \mathrm{~g}$ B.W + DMH $), G 3=G O S(84 \mathrm{mg} / 100$ g B.W + DMH $), G 4=G O S(107.14$ $\mathrm{mg} / 100$ g B.W+DMH), G5=basal diet alone. $\mathrm{a}=\mathrm{p}<0.05 \mathrm{v} / \mathrm{s} \quad \mathrm{Go}$, $\mathrm{b}=\mathrm{p}<0.05 \mathrm{v} / \mathrm{s}$ G1, c $=\mathrm{p}<0.05 \mathrm{v} / \mathrm{s} \mathrm{G} 2, \mathrm{~d}=\mathrm{p}<0.05 \mathrm{v} / \mathrm{s}$ G3, e $=\mathrm{p}<0.05 \mathrm{v} / \mathrm{s}$ $\mathrm{G} 4, \mathrm{f}=\mathrm{p}<0.05 \mathrm{v} / \mathrm{s}$ G5.

Urea and creatinine: Repeated mean values of serum urea and creatinine are expressed in Tables 1-3. Results of serum urea concentration for G0 (inulin+DMH) group were significant with respect to G1 (DMH treated group), G3 (84 mg/100 g B.W GOS $+\mathrm{DMH}$ ) and G5 (basal diet group).

\begin{tabular}{|l|l|}
\hline Groups $(\mathbf{n}=\mathbf{9})$ & Total ACF count \\
\hline G0 & $38.000 \pm 13.527^{*}$ \\
\hline G1 & $204.333 \pm 35.697$ \\
\hline G2 & $105.666 \pm 21.779$ \\
\hline
\end{tabular}


Citation: $\quad$ Naeem N, Raza S, Mubeen H, Siddiqui SA, Khokhar R (2018) Efficacy and Enzymatic Synthesis of Prebiotic Galacto-Oligosaccharides from Lactose Against Colon Carcinogenesis in Male Rats. J Bioprocess Biotech 8: 330. doi:10.4172/2155-9821.1000330

Page 4 of 5

\begin{tabular}{|l|l|}
\hline G3 & $88.333 \pm 19.553$ \\
\hline G4 & $43.666 \pm 14.011$ \\
\hline G5 & $0.000 \pm 0.000$ \\
\hline
\end{tabular}

Liver enzymes: Alkaline transaminase (ALT) results showed a significant difference for G0 (Inulin+DMH) group with respect to $\mathrm{DMH}$ treated group (G1) and all three galacto-oligosaccharide groups G2, G3 and G4. Among prebiotic groups, G2 and G3 came out to be significantly different with respect to G4 and non-significant with respect to each other. For Alkaline phosphatase (ALP), no significant

Table 2: Effect of prebiotic treatment on total number of aberrant crypt foci $(\mathrm{ACF})$ counts at 60 days.

Among galacto-oligosaccharide groups, results of G2 $(60 \mathrm{mg} / 100 \mathrm{~g}$ B.W GOS+DMH) group were significantly different with respect to G3 (84 mg/100 g B.W GOS+DMH) and G4 (107.14 mg/100 g B.W GOS difference was observed between Inulin+DMH group (G0) and any of three galacto-oligosaccharide groups G2, G3 and G4 but a significant difference with respect to $\mathrm{G} 1(\mathrm{DMH}+$ basal diet). Also, all three galacto-oligosaccharide groups were non-significantly different with respect to each other. $+\mathrm{DMH})$ groups. Results of serum creatinine concentration remained non-significant between all groups.

\begin{tabular}{|c|c|c|c|}
\hline \multicolumn{2}{|l|}{ Parameter } & Groups & Mean \pm SD \\
\hline \multirow[t]{12}{*}{ Serum liver function tests } & \multirow[t]{6}{*}{ Alanine transaminase (ALT) } & G0 & $35.00 \pm 1.00^{*}$ \\
\hline & & $\mathrm{G} 1$ & $57.333 \pm 2.516$ \\
\hline & & $\mathrm{G} 2$ & $40.00 \pm 2.00$ \\
\hline & & G3 & $38.66 \pm 2.51$ \\
\hline & & G4 & $44.33 \pm 0.57$ \\
\hline & & G5 & $38.33 \pm 3.05$ \\
\hline & \multirow[t]{6}{*}{ Alkaline phosphatase (ALP) } & Go & $220.00 \pm 10.00$ \\
\hline & & $\mathrm{G} 1$ & $310.00 \pm 20.00$ \\
\hline & & $\mathrm{G} 2$ & $254.66 \pm 8.962$ \\
\hline & & G3 & $238.33 \pm 7.637$ \\
\hline & & G4 & $200.33 \pm 9.504$ \\
\hline & & G5 & $112.66 \pm 11.06$ \\
\hline \multirow[t]{12}{*}{ Serum kidney function tests } & \multirow[t]{6}{*}{ Urea } & Go & $20.66 \pm 3.511$ \\
\hline & & G1 & $40.66 \pm 4.50$ \\
\hline & & G2 & $28.66 \pm 3.21$ \\
\hline & & G3 & $23.00 \pm 3.60$ \\
\hline & & G4 & $17.66 \pm 3.05$ \\
\hline & & G5 & $15.00 \pm 2.00$ \\
\hline & \multirow[t]{6}{*}{ Creatinine } & Go & $0.500 \pm 0.0100$ \\
\hline & & $\mathrm{G} 1$ & $0.633 \pm 0.577$ \\
\hline & & $\mathrm{G} 2$ & $0.600 \pm 0.100$ \\
\hline & & G3 & $0.466 \pm 0.057$ \\
\hline & & G4 & $0.400 \pm 0.00$ \\
\hline & & G5 & $0.333 \pm 0.577$ \\
\hline
\end{tabular}

Table 3: Effect of prebiotic treatment on serum kidney and liver function tests at 30 days of trial. ${ }^{*}$ Values are expressed as Mean $\pm \mathrm{SD}$. G0=Inulin (84 mg/100 g B.W+DMH), G1=basal diet+DMH, G2=GOS (60 mg/100 g B.W+DMH), G3=GOS (84 mg/100 g B.W+DMH), G4=GOS (107.14 $\mathrm{mg} / 100 \mathrm{~g}$ B.W $+\mathrm{DMH}), \mathrm{G} 5=$ basal diet alone. 
Citation: $\quad$ Naeem N, Raza S, Mubeen H, Siddiqui SA, Khokhar R (2018) Efficacy and Enzymatic Synthesis of Prebiotic Galacto-Oligosaccharides from Lactose Against Colon Carcinogenesis in Male Rats. J Bioprocess Biotech 8: 330. doi:10.4172/2155-9821.1000330

Page 5 of 5

\section{Discussion}

Lactulose, fructo-oligosaccharides (FOS), and galactooligosaccharides (GOS) are those prebiotics which currently fulfill the three criteria defined for prebiotics and they clearly increase the number of lactobacilli and bifidobacteria thereby altering the balance of large intestinal microbiota [11]. A positive association between reduction in experimental colon cancer and prebiotics, probiotics or synbiotics (combination of probiotics and prebiotics) has been observed [12]. Thus, in the present study, the protective effects of GOS against chemically induced colon cancer was investigated in male rats. Significantly reduced number of aberrant crypt foci (ACF) and tumors in colon were seen in those groups which were fed on GOS. These results are in concordance with previous studies suggesting that prebiotics have the capability of minimizing the occurrence and variety of colon cancers which are chemically induced [12]. An intervention study was carried out in pre-menopanusal women who were obese as well as dyslipidemia. According to this study, safe and recommended daily ingestion of GOS was suggested to be $14 \mathrm{~g}$ [3]. Findings from a placebo crossover study suggest that ingestion of $20 \mathrm{~g}$ of GOS daily has a positive effect on accelerating colonic transit time, when fed to healthy individuals [10]. There are numerous studies supporting the fact that GOS show modulating effects on tumor succession both in rat and human colon carcinogenesis. In another study, modulating effects of GOS consumption on cell propagation was investigated. Proliferation of tumor cells can lead to high risk of cancer [13]. During the development of tumors in colon, an increase in apoptosis (cell death) and cell proliferation has been observed. It is also reported that apoptosis inducing effect of synbiotic or prebiotic is more significant shortly after cancer induction as compared to in last phase of colon cancer [14]. There are various studies suggesting that fructooligossacharides or galacto-oligosaccharides are helpful in early biomarkers on colon carcinogenesis [15-17].

\section{Conclusion}

Animals belonging to prebiotic galactooligosaccharides groups (G2, G3 and G4) and inulin (Go) had significantly decreased ACF counts per colon as compared to controlled animals (G1 and G5). The body mass of animals belonging to galactooligosaccharides (Group G2, G3 and G4) increased significantly higher as compared to control group (G1). Effect of pre-biotic treatment had significant effects on organ weights; liver, heart, spleen as well as small intestine length.

\section{References}

1. Niva M (2007) All foods affect health: Understandings of functional foods and healthy eating among health-oriented Finns. Appetite 48: 384-393.
2. Grajek W, Olejnik A, Sip A (2005) Probiotics, prebiotics and antioxidants as functional foods. Acta Biochimica Polonica 52: 665-671.

3. Roberfroid MB (1998) Prebiotics and synbiotics: concepts and nutritional properties. Br J Nutr 80: S197-S202.

4. Gibson GR, Roberfroid MB (1995) Dietary modulation of the human colonic microbiota: Introducing the concept of prebiotics. J Nutr 125: 1401-1412.

5. Fanaro S, Marten B, Bagna R, Vigi V, Fabris C, et al. (2009) Galactooligosaccharides are bifidogenic and safe at weaning: A double-blind randomized Multicenter Study. J Pediatr Gastr Nutr 48: 82-88.

6. Shoaf K, Mulvey GL, Armstrong GD, Hutkins RW (2006) Prebiotic galacto-oligosaccharides reduce adherence of enteropathogenic Escherichia coli to tissue culture cells. Infect Immun 74: 6920-6928.

7. Dias MC, Spinardi Barbisan AL, Rodrigues MA, de Camargo JL, Terán E, et al. (2010) Effects of lycopene, synbiotic and their association on early biomarkers of rat colon carcinogenesis. Food Chem Toxicol 48: 772-780.

8. Dyer AR, Burdoc GA, Carabin IG, Hass MC, Boyce J, et al. (2008) In vitro and in vivo safety study of a proprietary whey extract. Food Chem Toxicol 46: 1659-1665.

9. Beutler E (1982) A Manual of biochemical methods. Grune and Stratton, New York, p: 137.

10. Bird RP (1995) Role of aberrant crypt foci in understanding the pathogenesis of colon cancer. Cancer Lett 93: 55-71.

11. Niittynen L, Kajander K, Korpela R (2007) Galacto-oligosaccharides and bowel function. Scand J Food Nutr 51: 62-66.

12. Verma A, Shukla G (2013) Administration of prebiotic inulin suppresses 1,2 dimethyl hydrazinedihydrochloride induced procarcinogenic biomarkers fecal enzymes and paraneoplastic lesions in early colon carcinogenesis in Sprague Dawley rats. J Functional Foods 5: 991-996.

13. Gouribebi S, Moro GE, Schmitt J, Tandoi L, Rizzardi S, et al. (2008) Early dietary intervention with a mixture of prebiotic oligosaccharides reduces the incidence of allergic manifestations and infections during the first two years of life. J Nutri 138: 1091-1095.

14. Caderni G, Bianchini F, Mancina A, Spagnesi MT, Dolara P, et al. (2007) Effect of prebiotics on the growth of aberrant crypt foci in the colon of rats treated with 1,2-dimethylhydrazine. Cancer Res 51: 3721-3725.

15. Bingham SA (1990) Mechanisms and experimental and epidemiological evidence relating dietary fiber (non-starch polysaccharides) and starch to protection against large bowel cancer. Proceedings Nutrion Society, pp: 153-171.

16. Bird RP (1998) Aberrant crypt foci system to study cancer preventive agents in the colon in Methods in Molecular Medicine: Tumor Marker Protocols pp: 465-474.

17. Roberfroid MB (2007) Prebiotics: The concept revisited. J Nutr 137: 830S-837S. 